\title{
Acaricidal and Insect Antifeedant Effects of Essential Oils From Selected Aromatic Plants and Their Main Components
}

Félix Valcárcel ${ }^{1}$, A. Sonia Olmeda ${ }^{2 *}$, Marta G. González ${ }^{1}$, Maria Fe Andrés $^{3}$,
Juliana Navarro-Rocha ${ }^{4}$ and Azucena González-Coloma ${ }^{3 *}$

${ }^{1}$ Grupo de Parasitología Animal, Departamento de Reproducción Animal, Instituto Nacional de Investigaciones Agrarias (INIA), Madrid, Spain, ${ }^{2}$ Departamento de Sanidad Animal, Facultad de Veterinaria, Universidad Complutense de Madrid (UCM), Madrid, Spain, ${ }^{3}$ Instituto de Ciencias Agrarias, Consejo Superior de Investigaciones Científicas (CSIC), Madrid, Spain, ${ }^{4}$ Unidad de Recursos Forestales, Centro de Investigación y Tecnología Agraria de Aragón (CITA), Zaragoza, Spain

This work has demonstrated the ixodicidal and insect antifeedant effects of essential oils from 14 experimentally cultivated aromatic plants. The strong ixodicidal and antifeedant oils corresponded to Thymus zygis, Thymus vulgaris, Satureja montana, Oreganum virens, and Mentha suaveolens. The moderately active oils were from Lavandula angustifolia, Mentha piperita, Mentha spicata, Artemisa herba-alba, and Rosmarinus officinalis. The most effective larvicidal and antifeedant compounds were piperitenone oxide, carvacrol, piperitenone, and thymol, explaining the effects of the most active essential oils. The rest of the tested compounds were not ixodicidal or antifeedant. Therefore, the activity of moderately active oils cannot be explained by their main components (linalyl acetate, linalool, menthone, menthol, limonene, camphor, 1,8-cineole, $p$-cymene, $\alpha$-pìnene, and carvone), suggesting synergistic effects. Considering the ixodicidal and antifeedant effects of these extracts, the plants have been ranked in relation to Thymus vulgare, a commercial biopesticide ingredient, for their potential as botanical pesticides. T. zygis, S. montana, and M. suaveolens ranked over T. vulgaris as ixodicidal agents and S. montana as insecticidal. Therefore, we propose the plant populations of $S$. montana, T. zygis, and $M$. suaveolens tested here for further development as biopesticide ingredients.

Keywords: aromatic plant, essential oil, ixodicidal, antifeedant, Hyalomma lusitanicum, Spodoptera littoralis, Myzus persicae, Rhopaslosiphum padi

\section{INTRODUCTION}

Food safety and environmental concerns related to the use of pesticides have resulted in more restricted regulatory frameworks worldwide, reducing the number of commercial products available for crop protection and other pest management sectors including the control of vectors of human and livestock diseases. Therefore, new safer and effective insecticides are needed. Botanical pesticides are emerging as a solution to meet part of the demand (Isman, 2020a). Essential oils (EOs) that are composed of volatile secondary metabolites, mostly terpenes (Bakkali et al., 2008), are among the most important extracts acting as botanical insecticides (Regnault-Roger et al., 2012; Pavela and Benelli, 2016), and some are being commercialized as commercial pesticide ingredients (Isman, 2020b). 
Arthropods, including economically important disease vectors and insect pests, are an important target of the biological effects of EOs (Ntalli et al., 2019; Isman, 2020a,b). Tick-borne diseases are a serious health and economic problem, responsible for over 100,000 cases of human diseases worldwide (de la Fuente et al., 2008) and billions of dollars in losses to the livestock industry (Lotfi and Karima, 2020). Additionally, ticks are in expansion due to climate change (Abbas et al., 2018). For example, Hyalomma ticks, vectors of the Crimean-Congo hemorrhagic fever virus, have spread from their original distribution (African and Mediterranean environments) to other European countries, becoming an increasing public health concern (Chitimia-Dobler et al., 2019; Hansford et al., 2019; Buczek et al., 2020; Grandi et al., 2020). For many years, tick control has been carried out with synthetic acaricides, leading to the appearance of resistance (reviewed by Abbas et al., 2014) and being harmful to the environment. Therefore, new effective and safer tick control agents are needed. In this context, EOs have been reported as being toxic and/or repellent to ticks (Benelli et al., 2016, 2017a; Benelli and Pavela, 2018; Salman et al., 2020).

Crop yield damages caused by pest infestations and pesticide use are significant (Oerke, 2006; Gregory et al., 2009) and increasing with global warming. Adaptation measures to increased pest damage related to global warming may involve greater use of pesticides with detrimental effects on health, environmental damage, and increased pesticide resistance (Deutsch et al., 2018). Some important crop pests include the Egyptian cotton leafworm, Spodoptera littoralis (Boisduval) (Lepidoptera: Noctuidae), a highly polyphagous insect labeled as an A2 quarantine pest by the OEPP/EPPO (2015) due to its host range (Alford, 2007) and distribution (Centre for Agricultural Bioscience International, 2020a). The green peach aphid, Myzus persicae Sulzer (Hemiptera: Aphididae), is the most economically important aphid crop pest worldwide (van Emden and Harrington, 2017) due to its distribution (Centre for Agricultural Bioscience International, 2020b), host range (Blackman and Eastop, 2000), mechanisms of plant damage, life cycle, and its ability to evolve resistance to insecticides (Bass et al., 2014). The bird cherry-oat aphid, Rhopalosiphum padi L., is a global pest of cereals (van Emden and Harrington, 2017) and a vector of yellow dwarf viruses that cause significant crop losses in cereals (Finlay and Luck, 2011). Many EOs are good insecticidal candidates because of their direct effects, biodegradability, and their low level of toxicity to mammals (Isman, 2020a,b).

The commercial production of a botanical insecticide depends on the sustainable production of plant biomass for extraction. Therefore, the domestication and cultivation of aromatic and medicinal plants (AMPs) for the production of EOs contributes to species conservation and provides sustainability of the production and lower variations in active ingredients. For example, a selected chemotype of wormwood, Artemisia absinthium (Asteraceae), that lacks the toxic terpene $\beta$-thujone but produces other novel terpenoids that are toxic and antifeedant to a range of pest insects has been domesticated for cultivation and registered as a new plant variety (Gonzalez-Coloma et al., 2017).
TABLE 1 | List of the plant species used and their origin (experimental field locations in Aragón, Spain, and UTM coordinates).

\begin{tabular}{|c|c|}
\hline Plant species & Origin \\
\hline Artemisia dracunculus L. & $\begin{array}{l}\text { Ejea de los Caballeros }\left(42^{\circ} 7^{\prime} 45^{\prime \prime} \mathrm{N},\right. \\
\left.1^{\circ} 8^{\prime} 15^{\prime \prime} \mathrm{W}\right)\end{array}$ \\
\hline Artemisia herba-alba Asso. & Villafranca $\left(41^{\circ} 34^{\prime} 28^{\prime \prime} \mathrm{N}, 0^{\circ} 39^{\prime} 01^{\prime \prime} \mathrm{W}\right)$ \\
\hline Hyssopus officinalis L. & Teruel $\left(40^{\circ} 20^{\prime} 37^{\prime \prime} \mathrm{N}, 1^{\circ} 06^{\prime} 26^{\prime \prime} \mathrm{W}\right)$ \\
\hline Lavandula angustifolia L. & $\begin{array}{l}\text { Ejea de los Caballeros }\left(42^{\circ} 7^{\prime} 45^{\prime \prime} \mathrm{N},\right. \\
\left.1^{\circ} 8^{\prime} 15^{\prime \prime} \mathrm{W}\right)\end{array}$ \\
\hline Mentha piperita L. & $\begin{array}{l}\text { La Alfranca }\left(41^{\circ} 36^{\prime} 22^{\prime \prime} \mathrm{N}, 0^{\circ} 45^{\prime} 22^{\prime \prime} \mathrm{O}\right) \mathrm{La} \\
\text { Alfranca }\left(41^{\circ} 36^{\prime} 22^{\prime \prime} \mathrm{N}, 0^{\circ} 45^{\prime} 22^{\prime \prime} \mathrm{O}\right)\end{array}$ \\
\hline \multicolumn{2}{|l|}{ Mentha spicata L. } \\
\hline Mentha suaveolens Ehrh. & $\begin{array}{l}\text { Ejea de los Caballeros }\left(42^{\circ} 7^{\prime} 45^{\prime \prime} \mathrm{N},\right. \\
\left.1^{\circ} 8^{\prime} 15^{\prime \prime} \mathrm{W}\right)\end{array}$ \\
\hline $\begin{array}{l}\text { Origanum vulgare subsp. virens } \\
\text { Hoffmanns and Link }\end{array}$ & Fabara $\left(41^{\circ} 10^{\prime} \mathrm{N}, 0^{\circ} 10^{\prime} \mathrm{E}\right)$ \\
\hline Rosmarinus officinalis L. & Villafranca $\left(41^{\circ} 34^{\prime} 28^{\prime \prime} \mathrm{N}, 0^{\circ} 39^{\prime} 01^{\prime \prime} \mathrm{W}\right)$ \\
\hline Satureja montana L. & $\begin{array}{l}\text { Ejea de los Caballeros }\left(42^{\circ} 7^{\prime} 45^{\prime \prime} \mathrm{N},\right. \\
\left.1^{\circ} 8^{\prime} 15^{\prime \prime} \mathrm{W}\right)\end{array}$ \\
\hline Tanacetum vulgare L. & $\begin{array}{l}\text { Ejea de los Caballeros }\left(42^{\circ} 7^{\prime} 45^{\prime \prime} \mathrm{N},\right. \\
\left.1^{\circ} 8^{\prime} 15^{\prime \prime} \mathrm{W}\right)\end{array}$ \\
\hline Thymus mastichina L. & $\begin{array}{l}\text { Moncayo-Trasobares }\left(41^{\circ} 39^{\prime} 49.43^{\prime \prime} \mathrm{N},\right. \\
\left.1^{\circ} 37^{\prime} 48.11^{\prime \prime} \mathrm{W}\right)\end{array}$ \\
\hline Thymus vulgaris $\mathrm{L}$. & Villarroya $\left(41^{\circ} 27^{\prime} 49^{\prime \prime} \mathrm{N}, 1^{\circ} 47^{\prime} 01^{\prime \prime} \mathrm{W}\right)$ \\
\hline Thymus zygis Loefl. ex L. & Aguarón $\left(41^{\circ} 20^{\prime} 20^{\prime \prime} \mathrm{N}, 1^{\circ} 16^{\prime} 11^{\prime \prime} \mathrm{W}\right)$ \\
\hline
\end{tabular}

As part of an ongoing project on the domestication and valorization of selected AMPs, plant species belonging to the genera Artemisia, Hyssopus, Lavandula, Mentha, Origanum, Rosmarinus, Satureja, Tanacetum, and Thymus have been experimentally cultivated at a small scale. These genera include species traditionally used in medicinal, food, and flavor applications due to their contents in bioactive EOs (Fathiazad and Hamedeyazdan, 2011; Chishti et al., 2013; Kumar and Tyagi, 2013; Tepe and Cilkiz, 2016; Aprotosoaie et al., 2017; Singh and Pandey, 2018; Borges et al., 2019; Li et al., 2019; Isman, 2020a,b).

In this work, essential oils from selected species of aromatic and medicinal plants cultivated experimentally (Table 1) have been evaluated against arthropods of importance in public health and animal and crop production: the tick (Hyalomma lusitanicum) and three insect pests (S. littoralis, M. persicae, and $R$. padi). Thymus vulgaris has been included in this study as a reference to compare the rest of the selected species because it is one of the most important aromatic plants grown worldwide (Southern and Central Europe, Southeast Asia, North America, and Africa), and it is an ingredient of botanical insecticides because of its thymol content (Pavela, 2016). Additionally, the composition of the most active EOs has been analyzed and the ixodicidal and insecticidal activities of their main components (Figure 1) tested.

\section{MATERIALS AND METHODS}

\section{Plant Material}

Fourteen plant species belonging to the families Asteraceae and Lamiaceae (Table 1) were selected for the study. The plants 


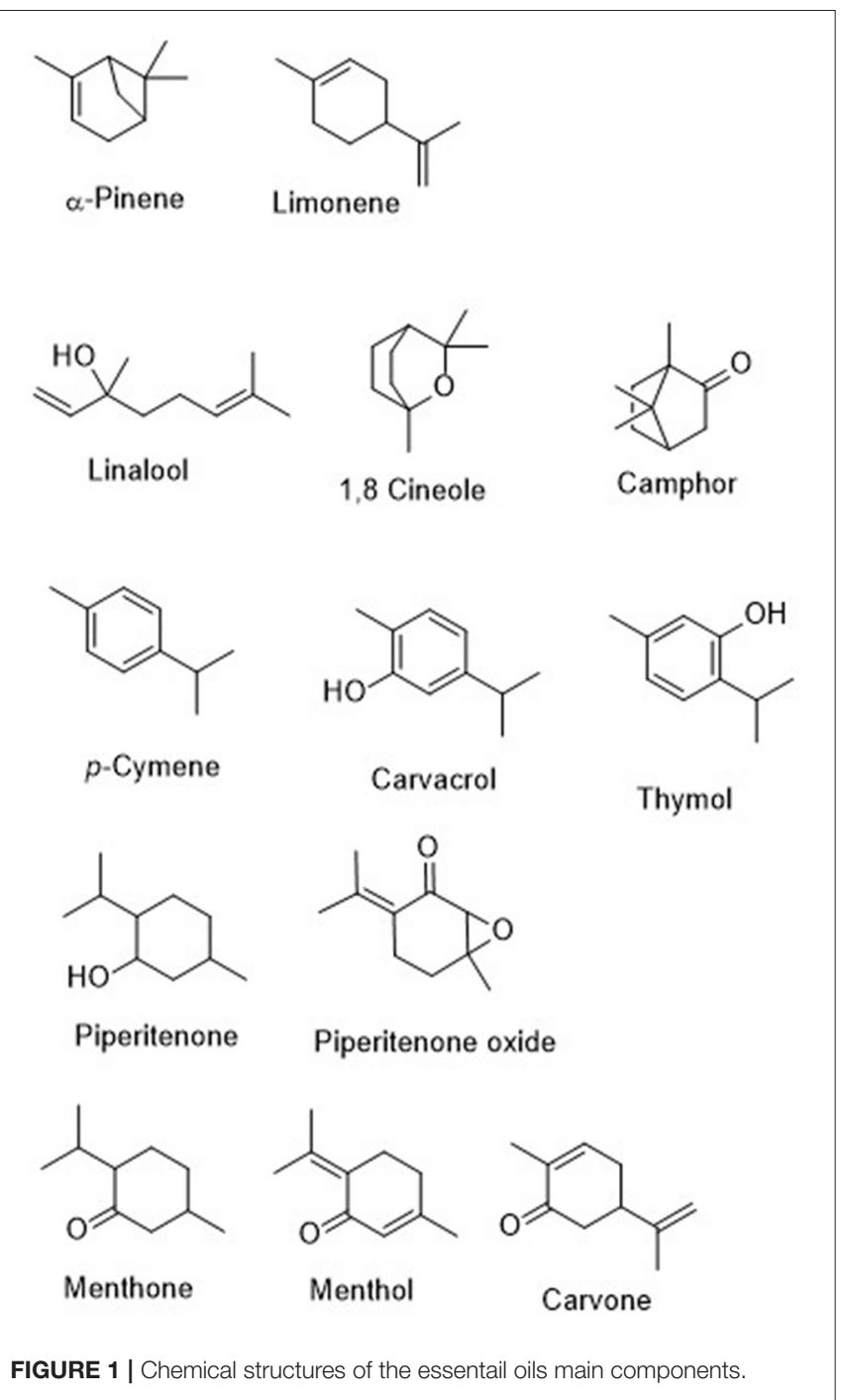

come from Spanish native flora and have been experimentally cultivated in several locations in Aragon (Spain) as described (Burillo, 2003; Burillo et al., 2017; Navarro-Rocha et al., 2020).

Aerial parts of these plants were collected at the flowering stage. EOs were obtained in the laboratory by Clevenger hydrodistillation (European Pharmacopoeia, 1975).

\section{Essential Oil Analysis}

The essential oils were analyzed by gas chromatographymass spectrometry (GC-MS) using a Shimadzu GC-2010 gas chromatograph coupled to a Shimadzu GCMS-QP2010 Ultra mass detector (electron ionization, $70 \mathrm{eV}$ ) and equipped with a $30-\mathrm{m} \times 0.25-\mathrm{mm}$ i.d. capillary column $(0.25 \mu \mathrm{m}$ film thickness $)$ Teknokroma TRB-5 (95\%) dimethyl-(5\%) diphenylpolisiloxane. The working conditions were as follows: split ratio, 20:1; injector temperature, $300^{\circ} \mathrm{C}$; temperature of the transfer line connected to the mass spectrometer, $250^{\circ} \mathrm{C}$; initial column temperature, $70^{\circ} \mathrm{C}$; then heated to $290^{\circ} \mathrm{C}$ at $6^{\circ} \mathrm{C} / \mathrm{min}$. The relative amounts of the individual components were calculated based on the peak area without using a correction factor. Electron ionization mass spectra, retention data, and the calculated linear retention indices (LRIs) were used to assess the identity of the compounds by comparing them with those of standards or those found in the Wiley 229 Mass Spectral Database.

\section{Ixodicidal Activity}

Hyalomma lusitanicum engorged females were collected from red deer in Ciudad Real (Central Spain) and maintained under laboratory conditions [ $22-24^{\circ} \mathrm{C}$ and $80 \%$ relative humidity (RH)] until oviposition and egg hatching.

Tick bioassays were performed according to Ruiz-Vásquez et al. (2017). Briefly, $50 \mu \mathrm{l}$ of the test solution was added to $25 \mathrm{mg}$ of powdered cellulose at different concentrations (initial concentration of 40 or $20 \mu \mathrm{g} / \mathrm{mg}$ for EOs or pure compounds, respectively) and the solvent was evaporated. The ticks and cellulose were then placed in laboratory glass tubes and carefully mixed by rotating the glass several times to ensure full tickcellulose contact. After mixing, the tubes were kept under laboratory conditions for $24 \mathrm{~h}$. For each test, three replicates with 20 active older than 6 weeks larvae. To validate the tests, three replicates of negative (cellulose, $25 \mathrm{mg}$ ) and positive (thymol, 20 $\mu \mathrm{g} / \mathrm{mg}$ ) controls were also used.

Ticks were considered dead when they could not move from one place to another. Dead ticks were counted after $24 \mathrm{~h}$ of contact with the treated cellulose at the laboratory conditions described using a binocular magnifying glass. The larvicidal activity data are presented as percent mortality corrected according to Schneider-Orelli's formula (Püntener, 1981). Effective lethal doses ( $\mathrm{LC}_{50}$ and $\mathrm{LC}_{90}$ ) were calculated by Probit analysis (1:2 serial dilutions to cover a range of activities between 100 and $<50 \%$ mortality with a minimum of three doses) (STATGRAPHICS Centurion XVI, version 16.1.02).

\section{Insect Antifeedant Activity}

Spodoptera littoralis, M. persicae, and $R$. padi colonies are maintained at ICA-CSIC, reared on artificial diet, bell pepper (Capsicum annuum) and barley (Hordeum vulgare) plants, respectively, and kept at $22 \pm 1^{\circ} \mathrm{C}$ and $>70 \% \mathrm{RH}$, with a photoperiod of $16: 8 \mathrm{~h}(\mathrm{~L} / \mathrm{D})$ in a custom-made walk-in growth chamber.

The bioassays were conducted as described (Navarro-Rocha et al., 2018). The upper surfaces of the C. annuum and H. vulgare leaf disks or fragments $\left(1.0 \mathrm{~cm}^{2}\right)$ were treated with $10 \mu \mathrm{l}$ of the test substance. The EOs and products were tested at an initial dose of 10 or $5 \mu \mathrm{g} / \mu \mathrm{l}\left(100\right.$ or $\left.50 \mu \mathrm{g} / \mathrm{cm}^{2}\right)$, respectively. Five to seven Petri dishes or 20 ventilated plastic boxes $(2 \times 2 \mathrm{~cm})$ with two sixth-instar S. littoralis larvae ( $\geq 24 \mathrm{~h}$ after molting) or 10 apterous aphid adults (24-48 h old) each were allowed to feed in a growth chamber (until 75\% larval consumption of the control disks or $24 \mathrm{~h}$ for aphids, environmental conditions as above). Each experiment was repeated twice. Feeding inhibition or aphid settling was calculated by measuring the disk surface consumption (digitalized with https://imagej.nih. gov/ij/) (Rueden et al., 2017) or by counting the number of aphids on each leaf fragment. Feeding/settling inhibition (\%FI or $\% \mathrm{SI})$ was calculated as $\% \mathrm{FI} / \mathrm{SI}=[1-(T / C) \times 100]$, where 
TABLE 2 | Larvicidal effects of the selected essential oils on Hyalomma lusitanicum.

\begin{tabular}{|c|c|c|c|}
\hline \multirow[t]{2}{*}{ Essential oil } & \multicolumn{3}{|c|}{ Hyalomma Iusitanicum } \\
\hline & $\%$ Mortalitya $(40 \mu \mathrm{g} / \mathrm{mg})$ & $\operatorname{LD}_{50}(C L)^{b}$ & $\operatorname{LD}_{90}(\mathrm{CL})^{\mathrm{b}}$ \\
\hline Artemisia dracunculus & $30.20 \pm 11.52$ & $>40$ & $>40$ \\
\hline Artemisia herba-alba & 100 & $20-40$ & $20-40$ \\
\hline Hyssopus officinalis & 0 & $>40$ & $>40$ \\
\hline Lavandula angustifolia & 100 & 16.06 (14.72-17.18) & $19.71(18.55-21.18)$ \\
\hline Mentha piperita & 100 & 22.96 (21.06-26.16) & 30.34 (26.9-37.64) \\
\hline Mentha suaveolens & 100 & $4.54(4.18-4.92)$ & $6.12(5.64-6.92)$ \\
\hline Mentha spicata & 100 & 23.58 (21.46-26.14) & $33.86(30.58-38.84)$ \\
\hline Origanum vulgare subsp.virens & 100 & $6.38(5.82-7.00)$ & $8.96(8.18-10.10)$ \\
\hline Rosmarinus officinalis & 100 & $\sim 10$ & $\sim 12$ \\
\hline Satureja montana & 100 & $4.68(4.14-5.24)$ & 8.33 (7.54-9.38) \\
\hline Tanacetum vulgare & $23.37 \pm 6.74$ & $>40$ & $>40$ \\
\hline Thymus mastichina & $47.69 \pm 20.50$ & $>40$ & $>40$ \\
\hline Thymus vulgaris & 100 & $5.52(4.42-6.36)$ & $9.52(8.46-11.36)$ \\
\hline Thymus zygis & 100 & $2.44(2.18-2.74)$ & $3.88(3.48-4.48)$ \\
\hline
\end{tabular}

a Values (in percent) are the means of three replicates corrected according to Schneider-Orelli's formula (Püntener, 1981).

${ }^{b}$ Lethal doses (upper-lower $95 \%$ confidence limits) calculated to give $50 \%\left(L D_{50}\right)$ or $90 \%\left(L D_{90}\right)$ mortality by Probit analysis.

$T$ and $C$ represent feeding/settling on the treated and control leaf disks, respectively. The antifeedant effects (\%FI/SI) were analyzed for significance by the non-parametric Wilcoxon paired signed-rank test comparing the consumption/settling between the treatment and control leaf disks. Extracts and compounds with an SI $>70 \%$ were further tested in a dose-response experiment (1:2 serial dilutions to cover a range of activities between 100 and $<50 \%$ feeding inhibition with a minimum of three doses) to calculate their relative potency $\left(\mathrm{EC}_{50}\right.$, the effective dose to give a $50 \%$ settling reduction) from the linear regression analysis (\%FI/SI on Log-dose, STATGRAPHICS Centurion XVI, version 16.1.02).

\section{RESULTS}

\section{Ixodicidal Effects}

Most of the EOs tested (75\%) gave significant ixodicidal activity against $H$. lusitanicum larvae (Table 2), which can be grouped into four categories as follows:

(1) Strong ixodicidal effects $\left(\mathrm{LC}_{50}<10 \mu \mathrm{g} / \mathrm{mg}\right.$ ): Thymus zygis (four doses tested, 100-46\% mortality), followed by Mentha suaveolens (five doses tested, 100-50\% mortality), Satureja montana (seven doses tested, 100-18\% mortality), T. vulgaris (four doses tested, 100-5\% mortality), and Origanum vulgare subsp. virens (six doses tested, 100-10\% mortality).

(2) Moderate ixodicidal effects $\left(\mathrm{LC}_{50}<16-28 \mu \mathrm{g} / \mathrm{mg}\right.$ ): Mentha piperita (three doses, 100-2\% mortality), Mentha spicata (three doses, 100-9\% mortality), Lavandula angustifolia (two doses, 92-2\% mortality), and Rosmarinus officinalis (two doses, 100-20\% mortality).

(3) Moderate-low ixodicidal effects $\left(\mathrm{LC}_{50}<20-40 \mu \mathrm{g} / \mathrm{mg}\right.$ ): Artemisia herba-alba, only toxic at the highest dose tested (40 $\mathrm{g} / \mathrm{mg}, 100 \%$ mortality).
(4) No ixodicidal effects ( $\mathrm{LC}_{50}>40 \mu \mathrm{g} / \mathrm{mg}$ ): Artemisia dranunculus, Hyssopus officinalis, Tanacetum vulgare, and Thymus mastichina.

\section{Antifeedant Effects}

Table 3 shows the insect antifeedant effects of the tested EOs. Overall, the herbivorous insects were less affected by these EOs than the tick (37 and 31\% EOs effective against S. littoralis and aphids, respectively).

Spodoptera littoralis feeding was strongly affected by S. montana (four doses, $\% \mathrm{FI}=90-5, \mathrm{EC}_{50}=39 \mu \mathrm{g} / \mathrm{cm}^{2}$ ), followed by M. piperita, M. spicata, T. vulgaris, T. zygis, and T. vulgare $(\% \mathrm{FI}=70-80)$.

Mentha persicae and $R$. padi were strongly affected by T. vulgaris (four doses, $\% \mathrm{SI}=81-10$ and $84-7, \mathrm{EC}_{50}=29$ and $49 \mu \mathrm{g} / \mathrm{cm}^{2}$, respectively) and S. montana (four doses, \%SI = $90-5, \mathrm{EC}_{50}=29 \mu \mathrm{g} / \mathrm{cm}^{2}$ ). M. suaveolens (three doses, \%SI = 92-20), O. vulgare subsp. virens (three doses, \%SI $=78-10$ ), and T. zygis (three doses, $\% \mathrm{SI}=89-15$ ) showed moderate effects on M. persicae $\left(\mathrm{EC}_{50}=35,34\right.$, and $45 \mu \mathrm{g} / \mathrm{cm}^{2}$, respectively). T. zygis, T. vulgare, and O. vulgare subsp. virens had low effects on $R$. padi $(\%$ SI $=65-70)$.

\section{Plant Species Ranking}

Considering the ixodicidal and antifeedant effects of the tested EOs, the plants have been ranked in relation to $T$. vulgaris (Table 4) for their potential as botanical pesticide ingredients. The ranking index has been established as [T. vulgaris $\mathrm{EC}_{50}$ value/ranked species $\mathrm{EC}_{50}$ value] for each test with significant effects (see Tables 2, 3).

Overall, considering the sum of all the indices, S. montana and $T$. zygis ranked over $T$. vulgaris (value $>4$ ). However, T. zygis, S. montana, and M. suaveolens ranked over T. vulgaris as 
TABLE 3 | Insect antifeedant effects of the selected essential oils.

\begin{tabular}{|c|c|c|c|}
\hline \multirow[t]{3}{*}{ Essential oil } & $\begin{array}{l}\text { Spodoptera } \\
\text { littoralis }\end{array}$ & $\begin{array}{c}\text { Myzus } \\
\text { persicae }\end{array}$ & $\begin{array}{c}\text { Rhopalosiphum } \\
\text { padi }\end{array}$ \\
\hline & $\% \mathrm{FI}^{\mathrm{a}}$ & \multicolumn{2}{|r|}{$\% \mathbf{S I}^{\mathrm{b}}$} \\
\hline & \multicolumn{3}{|c|}{$\mathrm{EC}_{50}(\mathrm{CL})^{\mathrm{c}}$} \\
\hline Artemisia dracunculus & $\begin{array}{c}53.4 \pm 11 \\
\sim 100\end{array}$ & $\begin{array}{c}21.8 \pm 6 \\
>100\end{array}$ & $\begin{array}{c}42.9 \pm 7 \\
>100\end{array}$ \\
\hline Artemisia herba-alba & $\begin{array}{c}30.2 \pm 10 \\
>100\end{array}$ & $\begin{array}{c}59.4 \pm 7 \\
>100\end{array}$ & $\begin{array}{c}31.5 \pm 7 \\
>100\end{array}$ \\
\hline Hyssopus officinalis & $\begin{array}{c}40.1 \pm 3 \\
>100\end{array}$ & $\begin{array}{c}41.2 \pm 8 \\
>100\end{array}$ & $\begin{array}{c}26.6 \pm 7 \\
>100\end{array}$ \\
\hline Lavandula angustifolia & $\begin{array}{c}54.8 \pm 11 \\
\sim 100\end{array}$ & $\begin{array}{c}31.0 \pm 8 \\
>100\end{array}$ & $\begin{array}{c}46.5 \pm 6 \\
>100\end{array}$ \\
\hline Mentha piperita & $\begin{array}{c}74.6 \pm 8^{*} \\
>70\end{array}$ & $\begin{array}{c}38.5 \pm 10 \\
>100\end{array}$ & $\begin{array}{c}33.2 \pm 8 \\
>100\end{array}$ \\
\hline Mentha spicata & $\begin{array}{c}72.84 \pm 12^{*} \\
>70\end{array}$ & $\begin{array}{c}56.7 \pm 8 \\
\sim 100\end{array}$ & $\begin{array}{c}17.8 \pm 5 \\
>100\end{array}$ \\
\hline Mentha suaveolens & $\begin{array}{c}71.1 \pm 14^{*} \\
>70\end{array}$ & $\begin{array}{c}92.1 \pm 3^{*} \\
35.0(31-39)\end{array}$ & $\begin{array}{c}48.5 \pm 7 \\
>100\end{array}$ \\
\hline Origanum vulgare subsp. virens & $\begin{array}{c}37.7 \pm 11 \\
>100\end{array}$ & $\begin{array}{c}78.0 \pm 7^{\star} \\
33.7(23-50)\end{array}$ & $\begin{array}{c}67.2 \pm 8^{\star} \\
>70\end{array}$ \\
\hline Rosmarinus officinalis & $\begin{array}{c}35.5 \pm 11 \\
>100\end{array}$ & $\begin{array}{c}51.4 \pm 6 \\
\sim 100\end{array}$ & $\begin{array}{c}19.9 \pm 5 \\
>100\end{array}$ \\
\hline Satureja montana & $\begin{array}{c}94.3 \pm 1^{*} \\
39.5(13-63)\end{array}$ & $\begin{array}{c}93.5 \pm 2^{*} \\
28.9(22-34)\end{array}$ & $\begin{array}{c}90.1 \pm 3^{\star} \\
29.2(20-38)\end{array}$ \\
\hline Tanacetum vulgare & $\begin{array}{c}68.2 \pm 10^{*} \\
>70\end{array}$ & $\begin{array}{c}51.8 \pm 7 \\
\sim 100\end{array}$ & $\begin{array}{c}68.1 \pm 6^{\star} \\
>70\end{array}$ \\
\hline Thymus mastichina & $\begin{array}{c}38.7 \pm 10 \\
>100\end{array}$ & $\begin{array}{c}33.5 \pm 9 \\
\sim 100\end{array}$ & $\begin{array}{l}8.7 \pm 5 \\
>100\end{array}$ \\
\hline Thymus vulgaris & $\begin{array}{c}74.9 \pm 12^{*} \\
>70\end{array}$ & $\begin{array}{c}80.7 \pm 6^{\star} \\
29.0(10-35)\end{array}$ & $\begin{array}{c}83.9 \pm 5^{\star} \\
49.0(40-50)\end{array}$ \\
\hline Thymus zygis & $\begin{array}{c}72.3 \pm 15^{\star} \\
>70\end{array}$ & $\begin{array}{c}89.3 \pm 5^{\star} \\
45.0(40-50)\end{array}$ & $\begin{array}{c}70.2 \pm 8^{\star} \\
>70\end{array}$ \\
\hline
\end{tabular}

a Percent feeding (FI) inhibition at a dose of $100 \mu \mathrm{g} / \mathrm{cm}^{2}$. Values are the means of five to seven replicates per dose.

Values with asterisk are significantly different according to Wilcoxon paired rank test $(P$ $<0.05)$.

${ }^{b}$ Percent setting (SI) inhibition at a dose of $100 \mu \mathrm{g} / \mathrm{cm}^{2}$. Values are the means of 20 replicates per dose.

${ }^{c} E C_{50}$ (95\% lower-upper confidence limits), concentration needed to produce $50 \%$ feeding/setting inhibition.

ixodicidal agents $(>1)$, and only $S$. montana ranked better than T. vulgaris against insects (Table 4).

\section{Essential Oil Composition}

Table 5 shows the main components (\% abundance >10) of the active EOs. The oils can be grouped according to their main components as follows: camphor/1,8-cineole $(+p$ cymene and $A$. herba-alba; $+\alpha$-pìnene and $R$. officinalis); carvacrol (S. montana); carvone/1,8-cineole ( $M$. spicata); $p$ cymene/carvacrol/linalool (O. vulgare subsp. virens); linalyl acetate/linalool (L. angustifolia); menthone/menthol/limonene
(M. piperita); piperitenone oxide/piperitenone (M. suaveolens); and thymol (T. zygis) ( + p-cymene and T. vulgaris $)$.

\section{Ixodicidal and Antifeedant Effects of EOs' Main Components}

Table 6 shows the ixodicidal effects of the selected individual components. Piperitenone oxide was the strongest acaricidal compound ( $\mathrm{LD}_{50-90}=0.9-1.1 \mu \mathrm{g} / \mathrm{mg}$ ), followed by carvacrol $\left(\mathrm{LD}_{50-90}=1.4-1.7 \mu \mathrm{g} / \mathrm{mg}\right)$, piperitenone $\left(\mathrm{LD}_{50-90}=1.8-2.2\right.$ $\mu \mathrm{g} / \mathrm{mg})$, and thymol ( $\left.\mathrm{LD}_{50-90}=2.9-6.2 \mu \mathrm{g} / \mathrm{mg}\right)$.

The antifeedant effects of the individual EO components are shown in Table 7. Piperitenone was the most effective antifeedant against $S$. littoralis $\left(\mathrm{EC}_{50}=1.4 \mu \mathrm{g} / \mathrm{cm}^{2}\right)$, followed by piperitenone oxide $\left(\mathrm{EC}_{50}=5 \mu \mathrm{g} / \mathrm{cm}^{2}\right)$, thymol $\left(\mathrm{EC}_{50}=\right.$ $\left.21 \mu \mathrm{g} / \mathrm{cm}^{2}\right)$, and $\alpha$-pinene with moderate-low effects $\left(\mathrm{EC}_{50}=\right.$ $\left.\sim 37 \mu \mathrm{g} / \mathrm{cm}^{2}\right)$. M. persicae strongly responded to thymol $\left(\mathrm{EC}_{50}\right.$ $\left.=7.6 \mu \mathrm{g} / \mathrm{cm}^{2}\right)$ and piperitenone oxide $\left(\mathrm{EC}_{50}=8.6 \mu \mathrm{g} / \mathrm{cm}^{2}\right)$, followed by carvacrol $\left(\mathrm{EC}_{50}=15 \mu \mathrm{g} / \mathrm{cm}^{2}\right)$ and menthone $\left(\mathrm{EC}_{50}\right.$ $\left.=\sim 34 \mu \mathrm{g} / \mathrm{cm}^{2}\right)$. R. padi was the least sensitive insect species and responded to carvacrol $\left(\mathrm{EC}_{50}=15 \mu \mathrm{g} / \mathrm{cm}^{2}\right)$, thymol $\left(\mathrm{EC}_{50}=19\right.$ $\left.\mu \mathrm{g} / \mathrm{cm}^{2}\right)$, and piperitenone oxide $\left(\mathrm{EC}_{50}=\sim 25 \mu \mathrm{g} / \mathrm{cm}^{2}\right)$.

\section{DISCUSSION}

This work has demonstrated the ixodicidal and insect antifeedant effects of EOs from experimentally cultivated AMPs. Furthermore, more EOs were ixodicidals than insect antifeedants, probably because of their different feeding ecologies (blood sucking vs. herbivores). Ticks are obligate hematophagous ectoparasites (Basu and Charles, 2017) and therefore have not evolved adaptations to plant secondary metabolites. On the other hand, insect herbivores have coevolved with plants and their chemical defenses/secondary metabolites (Maron et al., 2019). These differences in feeding adaptations could explain the selective toxicity of EOs toward the ticks observed here.

The EOs grouped as strong ixodicidal agents corresponded to T. zygis, T. vulgaris, S. montana, M. suaveolens, and Origanum virens. Similarly, the EOs grouped as strong antifeedants corresponded to S. montana, T. zygis, and T. vulgaris, followed by $O$. virens and $M$. suaveolens.

Thymus vulgaris EO, an ingredient of botanical pesticides (Pavela, 2016), has been included in this work as a reference for further species selection. In this work, the EO from T. vulgaris (thymol/ $p$-cymene) was the third most ixodicidal and the second most antifeedant against the insect species tested. The most common T. vulgaris chemotypes are thymol/carvacrol (György et al., 2020), which have reported ixodicidal effects including repellency against nymphs of Ixodes ricinus and adults of Dermacentor reticulatus (Štefanidesová et al., 2017; Goode et al., 2018), but not on its larvicidal effects against $H$. lusitanicum. EO from $T$. vulgaris has also been described as being insecticidal against several insect species, including $S$. littoralis, $M$. persicae (toxicity; Pavela, 2012; Ikbal and Pavela, 2019), and R. padi (antifeedant; Grul'ová et al., 2017). The EO from T. zygis (thymol) was the most effective ixodicidal agent tested here, with insect antifeedant effects similar to T. vulgaris. Previous 


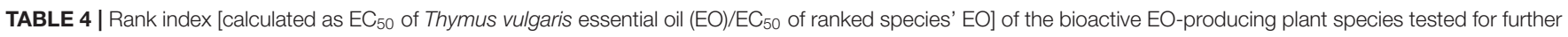
selection.

\begin{tabular}{|c|c|c|c|c|c|}
\hline Essential oil & Hyalomma lusitanicum & Spodoptera littoralis & Myzus persicae & Rhopas/osiphum padi & Total index \\
\hline Thymus vulgaris & 1 & 1 & 1 & 1 & 4 \\
\hline Satureja montana & 1.17 & 1.67 & 1 & 2.43 & 6.27 \\
\hline Thymus zygis & 2.25 & 0.95 & 0.64 & 0.69 & 4.53 \\
\hline Mentha suaveolens & 1.22 & 0.94 & 0.83 & & 2.99 \\
\hline Origanum vulgare subsp. virens & 0.86 & & 0.86 & 0.66 & 2.05 \\
\hline Mentha spicata & 0.23 & 1 & & & 1.23 \\
\hline Mentha piperita & 0.23 & 1 & & & 1.23 \\
\hline Rosmarinus officinalis & 0.55 & & & & 0.55 \\
\hline Lavandula angustifolia & 0.34 & & & & 0.34 \\
\hline
\end{tabular}

TABLE 5 | Main components of the active essential oils.

\begin{tabular}{|c|c|}
\hline Plant species & Compound (\% abundance) \\
\hline Artemisa herba-alba & Camphor (19), 1,8-cineole (12), p-cymene (8), borneol (1) \\
\hline $\begin{array}{l}\text { Lavandula } \\
\text { angustifolia }\end{array}$ & $\begin{array}{l}\text { Linalyl acetate (30), linalool (30), geranyl acetate (7), } \\
\text { terpineol (4), c-linalool oxide (3), } t \text {-linalool oxide (3), } \\
\text { caryophyllene oxide (3), neryl acetate (2) }\end{array}$ \\
\hline Mentha piperita & Menthone (41), menthol (31), limonene (13) \\
\hline Mentha spicata & Carvone (79),1,8-cineole (12), menthol (2) \\
\hline Mentha suaveolens & $\begin{array}{l}\text { Piperitenone oxide (37), piperitenone (21), limonene (7), } \\
\text { D-germacrone (7), } t \text {-caryophyllene (6) }\end{array}$ \\
\hline $\begin{array}{l}\text { Origanum vulgare } \\
\text { subsp. virens }\end{array}$ & $\begin{array}{l}\text { p-Cymene (30), carvacrol (17), linalool (14), } \alpha \text {-terpinene } \\
\text { (3), myrcene (2), } \beta \text {-caryophyllene (2) }\end{array}$ \\
\hline Rosmarinus officinalis & $\begin{array}{l}\text { Camphor (28), 1,8-cineole (22), } \alpha \text {-pinene (11), } \\
\text { endoborneol (6), camphene (6), verbenone (5) }\end{array}$ \\
\hline Satureja montana & $\begin{array}{l}\text { Carvacrol (76), p-cymene (2), borneol (2), thymoquinone } \\
\text { (1), 1-octen-3-ol (1) }\end{array}$ \\
\hline Thymus vulgaris & Thymol (49), $p$-cymene (29), $\gamma$-terpinene (7), carvacrol (4) \\
\hline Thymus zygis & Thymol (74), $p$-cymene (9), $\gamma$-terpinene (7), carvacrol (4) \\
\hline
\end{tabular}

reports showed that T. zygis EO (rich in thymol) was ovicidal, larvicidal, antifeedant, and repellent against the insect Plutella xylostella (Sangha et al., 2017), but this is the first report on its ixodicidal activity. T. zygis is distributed in the Iberian Peninsula and north of Africa (Morales Valverde, 1997), the thymol chemotype being of interest (Pérez-Sánchez et al., 2008). Therefore, the high content of thymol (75\%) and the effects on ticks of the EO from the T. zygis line tested here support further agronomic development.

The EO from S. montana (carvacrol) was the most effective insect antifeedant and the second most effective ixodicidal agent tested in this study. The essential oil of $S$. montana is characterized by carvacrol, thymol, $p$-cymene, and linalool (Velasco and Perez-Alonso, 1983; Silva et al., 2009; Dunkic et al., 2012). S. montana EO has reported repellence to Frankiniella occidentalis (Picard et al., 2012), is toxic against Leptinotarsa decemlineata larvae and adults (Usanmaz-Bozhuyuk and Kordali, 2018), larvicidal against Culex quinquefasciatus (Benelli et al., 2017b), and toxic to Drosophila suzukii adults (Park et al., 2016). The population of S. montana used in this work, rich in carvacrol,
TABLE 6 | Ixodicidal activity of the main components ( $\%$ abundance $\geq 10$ ) of the active essential oils on Hyalomma lusitanicum larvae.

\begin{tabular}{|c|c|c|c|}
\hline Compound & $\begin{array}{c}\% \text { Mortality }^{a} \\
(20 \mu \mathrm{g} / \mathrm{mg})\end{array}$ & $L_{50}(C L)^{b}$ & $L_{90}^{a}(C L)^{b}$ \\
\hline$\alpha$-Pinene & 0 & $>20$ & $>20$ \\
\hline Limonene & $6.87 \pm 1.84$ & $>20$ & $>20$ \\
\hline Linalool & $9.73 \pm 5.02$ & $>20$ & $>20$ \\
\hline 1,8-Cineole & $3.70 \pm 3.70$ & $>20$ & $>20$ \\
\hline Camphor & $15.60 \pm 4.73$ & $>20$ & $>20$ \\
\hline p-Cymene & $5.70 \pm 2.97$ & $>20$ & $>20$ \\
\hline Carvacrol & 100 & $1.42(1.34-1.54)$ & $1.76(1.62-1.92)$ \\
\hline Thymol & 100 & $2.94(2.08-3.54)$ & $6.16(5.30-7.84)$ \\
\hline Piperitenone & 100 & 1.77 (1.63-1.92) & $2.19(2.03-2.40)$ \\
\hline Piperitenone oxide & 100 & $0.88(0.81-0.96)$ & 1.09 (1.02-1.19) \\
\hline Menthone & $8.50 \pm 4.44$ & $>20$ & $>20$ \\
\hline Menthol & $31.4 \pm 13.6$ & $>20$ & $>20$ \\
\hline Carvone & $5.00 \pm 2.67$ & $>20$ & $>20$ \\
\hline
\end{tabular}

avalues (in percent) are the means of three replicates corrected according to SchneiderOrelli's formula (Püntener, 1981).

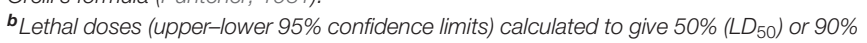
$\left(\left\llcorner D_{90}\right)\right.$ mortality by Probit analysis.

has already been included in an agronomic development program for the production of biopesticides (Navarro-Rocha et al., 2020). However, this is the first report on the ixodicidal activity of this EO.

The M. suaveolens population selected for this work was rich in piperitenone oxide/piperitenone. This EO was the second most effective ixodicidal extract tested here (more effective than T. vulgaris), along with S. montana, and showed stronger antifeedant effects against $M$. persicae than T. vulgaris. M. suaveolens is native of Africa, temperate Asia, and Europe (Abbaszadeh et al., 2009). There are three chemotypes described for M. suaveolens: pulegone, piperitenone oxide, and piperitenone oxide/piperitone oxide (Oumzil et al., 2002; BoŽović et al., 2015). Previously, M. suaveolens EOs (pulegone and menthone) showed ovicidal and larvicidal effects against the tick Hyalomma aegyptium (Laghzaoui et al., 2019). This species' EOs also have reported insecticidal 
TABLE 7 | Antifeedant activity of the main components (\% abundance $>10$ ) of the active essential oils on Spodoptera littoralis larvae, Myzus persicae, and Rhopaslosiphum padi apterous adults in choice tests.

\begin{tabular}{|c|c|c|c|}
\hline \multirow[t]{3}{*}{ Compound } & \multirow{2}{*}{$\frac{\text { S. litorallis }}{\% \mathrm{FI}^{\mathrm{a}}}$} & M. persicae & R. padi \\
\hline & & \multicolumn{2}{|c|}{$\% \mathrm{SI}^{\mathrm{b}}$} \\
\hline & & $\mathrm{EC}_{50}(\mathrm{CL})^{\mathrm{c}}$ & \\
\hline \multirow[t]{2}{*}{$\alpha$-Pinene } & $67.3 \pm 8.9$ & $53.9 \pm 10.2$ & $34.9 \pm 8.1$ \\
\hline & $\sim 37$ & $>50$ & $>50$ \\
\hline \multirow[t]{2}{*}{ Limonene } & $44.8 \pm 14.5$ & $29.3 \pm 7.7$ & $31.15 \pm 0.55$ \\
\hline & $>50$ & $>50$ & $>50$ \\
\hline \multirow[t]{2}{*}{ Linalool } & $45.3 \pm 7.2$ & $27.3 \pm 7.6$ & $48.4 \pm 8.3$ \\
\hline & $>50$ & $>50$ & $>50$ \\
\hline \multirow[t]{2}{*}{ 1,8 Cineole } & $36.0 \pm 8.7$ & $56.0 \pm 8.5$ & $21.9 \pm 7.2$ \\
\hline & $>50$ & $>50$ & $>50$ \\
\hline \multirow[t]{2}{*}{ Camphor } & $22.6 \pm 6.0$ & $37.6 \pm 7.0$ & $38.5 \pm 7.6$ \\
\hline & $>50$ & $>50$ & $>50$ \\
\hline \multirow[t]{2}{*}{ p-Cymene } & $8.61 \pm 6.09$ & $20.24 \pm 6.50$ & $35.0 \pm 7.5$ \\
\hline & $>50$ & $>50$ & $>50$ \\
\hline \multirow[t]{2}{*}{ Carvacrol } & $55.8 \pm 11.8$ & $86.4 \pm 3.2^{\star}$ & $90.6 \pm 5.3^{\star}$ \\
\hline & $\sim 50$ & 15.5 (11.1-18.8) & $14.6(11.7-18.2)$ \\
\hline \multirow[t]{2}{*}{ Thymol } & $52.4 \pm 10.1$ & $81.8 \pm 7.7^{\star}$ & $92.1 \pm 2.6^{\star}$ \\
\hline & $\sim 50$ & $7.6(4.1-8.7)$ & $18.6(4.1-23.3 .5)$ \\
\hline \multirow[t]{2}{*}{ Piperitenone } & $91.8 \pm 4.9^{*}$ & $56.2 \pm 2.4$ & $\mathrm{nt}$ \\
\hline & $1.45(0.2-9.9)$ & $\sim 50$ & \\
\hline \multirow[t]{2}{*}{ Piperitenone oxide } & $90.1 \pm 3.7^{\star}$ & $91.1 \pm 5.3^{*}$ & $75.0 \pm 6.5^{\star}$ \\
\hline & $5.0(1.8-13.5)$ & $8.6(3.0,24.5)$ & $\sim 25.0$ \\
\hline \multirow[t]{2}{*}{ Menthone } & $29.2 \pm 9.8$ & $72.8 \pm 9.2^{\star}$ & $61.6 \pm 6.7$ \\
\hline & $>50$ & $\sim 34$ & $>50$ \\
\hline \multirow[t]{2}{*}{ Menthol } & $35.6 \pm 14.3$ & $34.6 \pm 8.7$ & $45.4 \pm 8.7$ \\
\hline & $>50$ & $>50$ & $>50$ \\
\hline \multirow[t]{2}{*}{ Carvone } & $52.9 \pm 12.7$ & $31.0 \pm 9.8$ & $51.5 \pm 8.6$ \\
\hline & $\sim 50$ & $>50$ & $>50$ \\
\hline
\end{tabular}

apercent feeding (Fl) inhibition at a dose of $100 \mu \mathrm{g} / \mathrm{cm}^{2}$. Values are the means of five to seven replicates per dose. Values with asterisk are significantly different according to Wilcoxon paired rank test $(P<0.05)$.

${ }^{b}$ Percent setting (SI) inhibition at a dose of $100 \mu \mathrm{g} / \mathrm{cm}^{2}$. Values are the means of 20 replicates.

${ }^{c} E_{50}$ (95\% lower-upper confidence limits), concentration needed to produce $50 \%$ feeding/setting inhibition.

effects against stored-product pests such as Sitophilus oryzae (piperitenone oxide and piperitenone oxide/piperitenone chemotypes) (Zekri et al., 2013), Rizopertha dominica (piperitenone/pulegone/piperitone) (Benayad et al., 2012), and Triboleum castaneum (menthone/pulegone) (Kasrati et al., 2015) and larvicidal activity against C. quinquefasciatus (piperitenone oxide) (Pavela et al., 2014). Antifeedant effects against $L$. decemlineata and $M$. persicae have been reported for a piperitenone oxide/piperitone epoxide Mentha chemotype (Kimbaris et al., 2017). However, this is the first report on the ixodicidal effects of a piperitenone oxide/piperitenone M. suaveolens chemotype. The ixodicidal and antifeedant effects (stronger than those of T. vulgaris) of the M. suaveolens $\mathrm{EO}$ tested in this work support further agronomic development of this species for the production of biopesticides.

The chemotype of $O$. vulgare subsp. virens ( $p$-cymene, carvacrol, and linalool) tested here showed ixodicidal effects similar to T. vulgaris, but was less antifeedant, affecting only the aphid $M$. persicae. O. vulgare have reported toxic or repellent activities against $I$. ricinus (Soutar et al., 2019) and acute and fumigant toxicity against aphids including $M$. persicae (Ikbal and Pavela, 2019). However, this is the first report on the ixodicidal and aphid antifeedant effects of $O$. vulgare subsp. virens EO. O. vulgare is a widespread species native to the Mediterranean, the Euro-Siberian, and the Irano-Turanian regions and is one of the most traded and consumed spice plants (Lukas et al., 2015). O. vulgare subsp. virens is a heterogeneous subspecies characterized by essential oils rich in acyclic and/or cymyl compounds (Lukas et al., 2015). Since the effects of the EO from O. vulgare subsp. virens tested here were similar to these of $T$. vulgaris, its further development for the production of biopesticides against arthropods is not supported. However, we suggest the valorization of its essential oil production residues (biomass: hydrolate) as a potential source of biopesticidal ingredients.

The moderate ixodicidal EOs were from L. angustifolia (linalyl acetate/linalool), M. piperita (menthone/menthol), M. spicata (carvone/1,8-cineole), $R$. officinalis (camphor/1,8-cineole $/ \alpha$ pinene), and $A$. herba-alba (camphor/1,8-cineole/p-cymene). All these EOs were less effective than that of T. vulgaris.

The EO from L. angustifolia tested here (linalyl acetate/linalool) showed moderate ixodicidal effects against $H$. luxitanicum larvae, lower than the effects of T. vulgaris, without significant insect antifeedant effects. Previous reports have shown interference with the host-seeking behaviors of H. marginatum and D. reticulatus for this species' EO (Mkolo and Magano, 2007; Štefanidesová et al., 2017) and toxicity to Rhipicephalus (Boophilus) annulatus (Pirali-Kheirabadi and Teixeira da Silva, 2010) for this species' EO. Additionally, a similar EO from the hybrid Lavandula $\times$ intermedia (rich in linalyl acetate and linalool) was also toxic to $H$. lusitanicum larvae and moderately antifeedant to S. littoralis (Ortiz de Elguea-Culebras et al., 2018). L. angustifolia, distributed in the sub-Mediterranean region, has a great economic importance in perfumery, cosmetics, food, pharmaceutical industries, and aromatherapy (Demasi et al., 2018). However, our results do not support its agronomic production as a biopesticide when compared to $T$. vulgaris, but suggest the valorization of its essential oil production residues (biomass: hydrolate) as a source of biopesticidal ingredients.

M. piperita (menthone/menthol) was moderately ixodicidal against $H$. lusitanicum and showed moderate antifeedant effects against $S$. littoralis. In previous works, $M$. piperita EO showed moderate repellency against adults of $D$. reticulatus (Štefanidesová et al., 2017), larvicidal effects against $R$. microplus (de Souza Chagas et al., 2016), and toxicity against aphids including M. persicae (Ikbal and Pavela, 2019). M. spicata (carvone/1,8-cineole) also had moderate larvicidal effects against $H$. lusitanicum and moderate-low antifeedant effects on S. littoralis. M. spicata EO has been reported as a moderate 
repellent against adults of D. reticulatus (Štefanidesová et al., 2017) and toxic to stored-product pests (Irfan et al., 2009; Kedia et al., 2014; Eliopoulos et al., 2015; Nubia et al., 2016), L. decemlineata (Saroukolai et al., 2014), and S. littoralis (Pavela, 2005), while a carvone/limonene chemotype of $M$. spicata was not antifeedant or toxic to S. littoralis, M. persicae, and $R$. padi (Santana et al., 2014). Mentha oils are used commercially as biopesticide ingredients because of their various effects against insects, the most commercialized being the Mentha species spearmint (M. spicata), peppermint (M. piperita), and $M$. arvensis (Singh and Pandey, 2018). Since our results showed lower effects than T. vulgaris, we suggest the valorization of their commercial essential oil production residues (biomass: hydrolate) as an additional source of biopesticidal ingredients.

Rosmarinus officinalis (camphor/1,8-cineole/ $\alpha$-pinene) showed moderate ixodicidal effects in this work. Previous reports have shown a moderate post-ingestive toxicity to $S$. littoralis for a similar R. officinalis EO (Santana et al., 2014). R. officinalis EOs rich in 1,8-cineole were toxic to larvae of Hyalomma scupense (Djebir et al., 2019) and I. ricinus nymphs (Elmhalli et al., 2019), while an EO rich in $\alpha$-pinene showed low-moderate toxicity against larvae of $R$. (B.) microplus (Martinez-Velazquez et al., 2011). This plant is cultivated worldwide as a food flavoring and preservative due to its antioxidant and antimicrobial potential (Borges et al., 2019). Our results showed lower effects for R. officinalis than T. vulgaris. However, being a commercial plant available worldwide, we suggest the valorization of its essential oil production residues (biomass: hydrolate) as a source of biopesticidal ingredients.

The chemotype of $A$. herba-alba (camphor/1,8-cineole/pcymene) tested in this work showed low-moderate larvicidal effects against $H$. lusitanicum. A. herba-alba is a medicinal and aromatic shrub that grows wild in arid areas of the Mediterranean Basin, being abundant in the Iberian Peninsula (Mohamed et al., 2010), showing chemical diversity (Salido et al., 2004). An A. herba-alba EO rich in piperitone showed repellency against $I$. ricinus nymphs (El-Seedi et al., 2017), and a thujone/camphor chemotype was antifeedant and moderately toxic to S. littoralis (Santana et al., 2014). Our results showed lower effects for a camphor/1,8-cineole $A$. herba-alba chemotype than those reported or $T$. vulgaris. Given the chemical diversity of $A$. herba-alba wild populations, we suggest further research on chemotype-bioactivity correlations for this plant species prior to its selection for agronomical development.

Considering the plant species' rank based on the ixodicidal and antifeedant effects of their EOs, we propose the plant populations of S. montana, T. zygis, and M. suaveolens tested here for further agronomical development as biopesticide ingredients for the control of ticks and insects. These EOs (S. montana, T. zygis, and M. suaveolens) have additional biopesticidal effects such as strong nematicidal action against root-knot nematodes (Meloidogyne javanica), with S. montana being the most effective $\left(\mathrm{LC}_{50}=0.041 \mu \mathrm{g} / \mu \mathrm{l}\right)$ (Andrés et al., 2012).

To further understand the effects of the active EOs, their main components (Figure 1) were also tested against the selected targets. The most effective larvicidal and antifeedant compounds were piperitenone oxide, carvacrol, piperitenone, and thymol.
The activity of piperitenone oxide and piperitenone explained the effects of $M$. suaveolens EO. Thymol explained the effects of the EOs from T. zygis and T. vulgaris, while carvacrol was responsible for the effects of $S$. montana and $O$. vulgare subsp. virens.

These compounds have reported ixodicidal and/or insecticidal effects. Piperitenone epoxide and piperitenone showed strong larvicidal and repellent effects against Aedes albopictus (Giatropoulos et al., 2018). Piperitenone was antifeedant to L. decemlineata and S. littoralis (Kimbaris et al., 2017). However, there are no reports on the acaricidal effects of these compounds. Thymol was larvicidal to H. lusitanicum (Navarro-Rocha et al., 2018), and carvacrol was toxic to Rhipicephalus turanicus (Coskun et al., 2008) and moderately toxic to Hyalomma marginatum adults (Cetin et al., 2010). These compounds were repellent to Amblyomma americanum (Carroll et al., 2017) and showed strong toxicity against $I$. ricinus larvae and repellency against $I$. ricinus larvae and $A$. americanum nymphs (Carroll et al., 2017; Tabari et al., 2017). Carvacrol and thymol also have reported behavioral and toxic effects against several insect species, including the ones targeted here. Specifically, thymol was antifeedant to $M$. persicae (Navarro-Rocha et al., 2018). Thymol and carvacrol were antifeedant to $S$. littoralis fourth-instar larvae (Pavela, 2011) and affected the olfactory sensilla of female S. littoralis adults (Anderson et al., 1993). Additionally, carvacrol and thymol showed acute toxicity to $S$. littoralis third-instar larvae (Pavela, 2014), and carvacrol was toxic to $M$. persicae (Petrakis et al., 2014).

The rest of the tested compounds were not ixodicidal or antifeedant. Therefore, the activity of the moderately active EOs (L. angustifolia, M. piperita, M. spicata, R. officinalis, and A. herba-alba) cannot be explained by their main components (linalyl acetate, linalool, menthone, menthol, limonene, camphor, 1,8-cineole, $p$-cymene, $\alpha$-pìnene, and carvone), suggesting synergistic effects. $p$-Cymene was among the most frequent synergists found, interacting with 22 terpenes commonly present in EOs (Pavela et al., 2014). Therefore, synergistic interactions among EO components could explain their ixodicidal effects.

\section{CONCLUSION}

This work has demonstrated the ixodicidal and insect antifeedant effects of EOs from experimentally cultivated AMPs. The EOs grouped as strong ixodicidal agents corresponded to T. zygis, T. vulgaris, S. montana, M. suaveolens, and O. vulgare subsp. virens. Similarly, the EOs grouped as strong antifeedants corresponded to S. montana, T. zygis, and T. vulgaris, followed by $O$. vulgare subsp. virens and $M$. suaveolens. The moderate ixodicidal EOs were from L. angustifolia, M. piperita, M. spicata, A. herba-alba, and R. officinalis.

The most effective larvicidal and antifeedant compounds were piperitenone oxide, carvacrol, piperitenone, and thymol, explaining the effects of $M$. suaveolens, T. zygis, T. vulgaris, $S$. montana, and O. vulgare subsp. virens EOs. The rest of the tested compounds were not ixodicidal or antifeedant. Therefore, the activity of the moderately active EOs (L. angustifolia, 
M. piperita, A. herba-alba, R. officinalis, and M. spicata) cannot be explained by their main components (linalyl acetate, linalool, menthone, menthol, limonene, camphor, 1,8-cineole, $p$-cymene, $\alpha$-pìnene, and carvone), suggesting synergistic effects.

T. zygis, S. montana, and M. suaveolens were better ixodicidals and $S$. montana was a better antifeedant than $T$. vulgaris. Therefore, we propose the plant populations of S. montana, T. zygis, and M. suaveolens tested here for further development as biopesticide ingredients for the control of ticks and insect pests.

\section{DATA AVAILABILITY STATEMENT}

The raw data supporting the conclusions of this article will be made available by the authors, without undue reservation.

\section{AUTHOR CONTRIBUTIONS}

AG-C conceptualized the study. AG-C, AO, MG, JN-R, and FV curated the data. MG, FV, and MA did the formal analysis. AG-C, $\mathrm{AO}$, and FV helped with funding acquisition and resources. MG, JN-R, FV, AO, MA, and AG-C did the investigation. AO, FV, $\mathrm{AG}-\mathrm{C}$, and MA helped with the methodology. FV and AG-C

\section{REFERENCES}

Abbas, A., Abbas, R. Z., Zahib Iqbal, S. M., Khan, M. K., Saleemi, M. K., Raza, M. A., et al. (2018). Acaricidal and insecticidal effects of essential oils against ectoparasites of veterinary importance. Bol. Latinoam. Caribe Plant Med. Aromat. 17, 441-452.

Abbas, R. Z., Zaman, M. A., Colwell, D. D., Gilleard, J., and Iqbal, Z. (2014). Acaricide resistance in cattle ticks and approaches to its management: the state of play. Vet. Parasitol. 203, 6-20. doi: 10.1016/j.vetpar.2014.03.006

Abbaszadeh, B., Valadabadi, S. A., Farahani, H. A., and Darvishi, H. H. (2009). Studying of essential oil variations in leaves of Mentha species. Afr. J. Plant Sci. 3, 217-221.

Alford, D. V. (2007). Pests of Fruit Crops. Boston, MA: Academic Press. doi: $10.1201 / \mathrm{b} 15135$

Anderson, P., Hilker, M., Hansson, B. S., Bombosch, S., Klein, B., and Schildknecht, H. (1993). Oviposition deterring components in larval frass of Spodoptera littoralis (Boisd.) (Lepidoptera: Noctuidae): a behavioural and electrophysiological evaluation. J. Insect Physiol. 39, 129-137. doi: 10.1016/0022-1910(93)90104-Y

Andrés, M. F., González-Coloma, A., Sanz, J., Burillo, J., and Sainz, P. (2012). Nematicidal activity of essential oils: a review. Phytochem. Rev. 11, 371-390. doi: 10.1007/s11101-012-9263-3

Aprotosoaie, A. C., Gille, E., Trifan, A., Luca, V. S., and Miron, A. (2017). Essential oils of Lavandula genus: a systematic review of their chemistry. Phytochem. Rev. 16, 761-799. doi: 10.1007/s11101-017-9517-1

Bakkali, F., Averbeck, S., Averbeck, D., and Idaomar, M. (2008). Biological effects of essential oils. Food Chem. Toxicol. 46, 446-475. doi: 10.1016/j.fct.2007.09.106

Bass, C., Puinean, A. M., Zimmer, C. T., Denholm, I., Field, L. M., Foster, S. P., et al. (2014). The evolution of insecticide resistance in the peach potato aphid, Myzus persicae, Insect Biochem. Mol. Biol. 51, 41-51. doi: 10.1016/j.ibmb.2014.05.003

Basu, A. K., and Charles, R. A. (2017). "Chapter 1 - a general account of ticks," in Ticks of Trinidad and Tobago - An Overview, eds A. K. Basu and R. A. Charles (Boston, MA: Academic Press), 1-33. doi: 10.1016/B978-0-12-809744-1.00001-3

Benayad, N., Ebrahim, W., Hakiki, A., and Mosaddak, M. (2012). Chemical characterization and insecticidal evaluation of the essential oil of Mentha wrote the original draft. AG-C, AO, MA, FV, and JN-R did the writing-review and editing. All authors have read and agreed to the published version of the manuscript.

\section{FUNDING}

This work has been supported by grants from ERASMUS+ no. 2018-1-FR01-KA202-047892, Biocontrol E Training (BET), and ERASMUS+ 600873-EPP-1-2018-1-ES-EPPKA2. European Hub on New Challenges in the Field of Essential Oils (EOHUB), PID2019-106222RB-C31, MCI, Spain, RTA 2014-00080-0000, Spain, and the $\mathrm{R}+\mathrm{D}$ contracts CSIC-Villamagna Estudio Biodirigido de Extractos Ixodicidas and UCM-Villamagna SL Investigación en torno al control de las garrapatas y al estado sanitario de la fauna silvestre.

\section{ACKNOWLEDGMENTS}

We gratefully acknowledge His Grace the Duke of Westminster and J.M. Tercero (Finca La Garganta, Villamagna) for tick collection and logistic support. We also acknowledge $\mathrm{R}$. Muñoz, V. Morales-Sánchez, E. Moreno (ICA-CSIC), J. Burillo (CITA), María Sánchez (UAX), and Julia Gonzalez (INIA) for technical support. suaveolens Ehrh. and Mentha pulegium L. growing in Morocco. Food Ind. $13,27-32$.

Benelli, G., Maggi, F., Romano, D., Stefanini, C., Vaseeharan, B., Kumar, S., et al. (2017a). Nanoparticles as effective acaricides against ticks - a review. Ticks Tick-Borne Dis. 8, 821-826. doi: 10.1016/j.ttbdis.2017.08.004

Benelli, G., and Pavela, R. (2018). Repellence of essential oils and selected compounds against ticks-a systematic review. Acta Trop. 179, 47-54. doi: 10.1016/j.actatropica.2017.12.025

Benelli, G., Pavela, R., Canale, A., Cianfaglione, K., Ciaschetti, G., Conti, F., et al. (2017b). Acute larvicidal toxicity of five essential oils (Pinus nigra, Hyssopus officinalis, Satureja montana, Aloysia citrodora and Pelargonium graveolens) against the filariasis vector Culex quinquefasciatus: synergistic and antagonistic effects. Parasitol. Int. 66, 166-171. doi: 10.1016/j.parint.2017. 01.012

Benelli, G., Pavela, R., Canale, A., and Mehlhorn, H. (2016). Tick repellents and acaricides of botanical origin: a green roadmap to control tick-borne diseases? Parasitol. Res. 115, 2545-2560. doi: 10.1007/s00436-016-5095-1

Blackman, R. L., and Eastop, V. F. (2000). Aphids on the World's Crops, An Identification and Information Guide, 2nd Edn. Chichester, UK: John Wiley and Sons Ltd.

Borges, R. S., Ortiz, B. L. S., Pereira, A. C. M., Keita, H., and Carvalho, J. C. T. (2019). Rosmarinus officinalis essential oil: a review of its phytochemistry, antiinflammatory activity, and mechanisms of action involved. J. Ethnopharmacol. 30, 29-45. doi: 10.1016/j.jep.2018.09.038

BoŽović, M., Pirolli, A., and Ragno, R. (2015). Mentha suaveolens Ehrh. (Lamiaceae) essential oil and its main constituent piperitenone oxide: biological activities and chemistry. Molecules 20, 8605-8633. doi: 10.3390/molecules20058605

Buczek, A. M., Buczek, W., Buczek, A., and Bartosik, K. (2020). The potential role of migratory birds in the rapid spread of ticks and tick-borne pathogens in the changing climatic and environmental conditions in Europe. Int. J. Environ. Res. Public Health 17:2117. doi: 10.3390/ijerph17062117

Burillo, J. (2003). Investigación y experimentación de plantas aromáticas y medicinales en Aragón: cultivo, transformación y analítica. Zaragoza: Gobierno de Aragón. Departamento de Agricultura, 262. Available online at: http://hdl. handle.net/10532/1757 (accessed February 17, 2021). 
Burillo, J., Gonzalez-Coloma, A., Tapia, J., and Navarro-Rocha, J. (2017). "Principles of medicinal and aromatic plant (MAPs) propagation, production and collection of Artemisia absinthium L." in Frontiers in Horticulture Medicinal and Aromatic Plants: The Basics of Industrial Application Vol. 1, ed M. P. Arraiza (Sharjah: Bentham Science), 134-162. doi: $10.2174 / 9781681085500117010013$

Carroll, J. F., Demirci, B., Kramer, M., Bernier, U. R., Agramonte, N. M., Baser, K. H. C., et al. (2017). Repellency of the Origanum onites L. essential oil and constituents to the lone star tick and yellow fever mosquito. Nat. Prod. Res. 31, 2192-2197. doi: 10.1080/14786419.2017.1280485

Centre for Agricultural Bioscience International (2020a). Available online at: https://www.cabi.org/isc/datasheet/51070 (accessed May 20, 2020).

Centre for Agricultural Bioscience International (2020b). Available online at: https://www.cabi.org/isc/datasheet/35642 (accessed May 20, 2020).

Cetin, H., Cilek, J. E., Oz, E., Aydin, L., Deveci, O., and Yanikoglu, A. (2010). Acaricidal activity of Satureja thymbra L. essential oil and its major components, carvacrol and $\gamma$-terpinene against adult Hyalomma marginatum (Acari: Ixodidae). Vet. Parasitol. 170, 287-290. doi: 10.1016/j.vetpar.2010.02.031

Chishti, S., Kaloo, Z. A., and Sultan, P. (2013). Medicinal importance of genus Origanum: a review. J. Pharmacogn. Phytother. 5, 170-177. doi: 10.5897/JPP2013.0285

Chitimia-Dobler, L., Schaper, S., Rieß, R., Bitterwolf, K., Frangoulidis, D., Bestehorn, M., et al. (2019). Imported Hyalomma ticks in Germany in 2018. Parasites Vectors 12:134. doi: 10.1186/s13071-019-3380-4

Coskun, S., Girisgin, O., Kürkcüoglu, M., Malyer, H., Girisgin, A. O., and Kirimer, N. (2008). Acaricidal efficacy of Origanum onites L. essential oil against Rhipicephalus turanicus (Ixodidae). Parasitol. Res. 103, 259-261. doi: 10.1007/s00436-008-0956-x

de la Fuente, J., Estrada-Peña, A., Venzal, J., Kocan, K., and Sonenshine, D. (2008). Overview: ticks as vectors of pathogens that cause disease in humans and animals. Front. Biosci. 13, 6938-6946. doi: 10.2741/3200

de Souza Chagas, A. C., de Sena Oliveira, M. C., Giglioti, R., Santana, R. C. M., Bizzo, H. R., Gama, P. E., et al. (2016). Efficacy of 11 Brazilian essential oils on lethality of the cattle tick Rhipicephalus (Boophilus) microplus. Ticks Tick-borne Dis. 7, 427-432. doi: 10.1016/j.ttbdis.2016.01.001

Demasi, S., Caser, M., Lonati, M., Cioni, P. L., Pistelli, L., Najar, B., et al. (2018). Latitude and altitude influence secondary metabolite production in peripheral alpine populations of the mediterranean species lavandula angustifolia mill. Front. Plant Sci. 9:983. doi: 10.3389/fpls.2018.00983

Deutsch, C. A., Tewksbury, J. J., Tigchelaar, M., Battisti, D. S., Merrill, S. C., Huey, R. B., et al. (2018). Increase in crop losses to insect pests in a warming climate. Science 361, 916-919. doi: 10.1126/science.aat3466

Djebir, S., Ksouri, S., Trigui, M., Tounsi, S., Boumaaza, A., Hadef, Y., et al. (2019). Chemical composition and acaricidal activity of the essential oils of some plant species of lamiaceae and myrtaceae against the vector of tropical bovine theileriosis: Hyalomma scupense (syn. Hyalomma detritum). Biomed. Res. Int. 2019:7805467. doi: 10.1155/2019/7805467

Dunkic, V., Kremer, D., Muller, I. D., Stabentheiner, E., Kuzmić, S., Jurišić Grubešić, R., et al. (2012). Chemotaxonomic and micromorphological traits of Satureja montana L. and S. subspicata Vis. (Lamiaceae). Chem. Biodivers. 9, 2825-2842. doi: $10.1002 / \mathrm{cbdv} .201100376$

Eliopoulos, P. A., Hassiotis, C. N., and Andreadis, S. S. (2015). Fumigant toxicity of essential oils from basil and spearmint against two major pyralid pests of stored products. J. Econ. Entomol. 108, 805-810. doi: 10.1093/jee/tov029

Elmhalli, F., Garboui, S. S., Borg-Karlson, A. K., Mozuraitis, R., Baldauf, S. L., and Grandi, G. (2019). The repellency and toxicity effects of essential oils from the Libyan plants Salvadora persica and Rosmarinus officinalis against nymphs of Ixodes ricinus. Exp. Appl. Acarol. 77, 585-599. doi: 10.1007/s10493-019-00373-5

El-Seedi, H. R., Azeem, M., Khalil, N. S., Sakr, H. H., Khalifa, S. A. M., Awang, K., et al. (2017). Essential oils of aromatic Egyptian plants repel nymphs of the tick Ixodes ricinus (Acari: Ixodidae). Exp. Appl. Acarol. 73, 139-157. doi: 10.1007/s10493-017-0165-3

European Pharmacopoeia (1975). European Pharmacopoeia, Vol. 3. Maisonneuve SA, Sainte Ruffine: European Pharmacopoeia, 68.

Fathiazad, F., and Hamedeyazdan, S. (2011). Review on Hyssopus officinalis L.: composition and biological activities. Afr. J. Pharmacy Pharmacol. 5, 1959-1966. doi: 10.5897/AJPP11.527
Finlay, K. J., and Luck, J. E. (2011). Response of the bird cherry-oat aphid (Rhopalosiphum padi) to climate change in relation to its pest status, vectoring potential and function in a crop-vector-virus pathosystem. Agric. Ecosyst. Environ. 144, 405-421. doi: 10.1016/j.agee.2011.08.011

Giatropoulos, A., Kimbaris, A., Michaelakis, A., Papachristos, D. P., Polissiou, M. G., and Emmanouel, N. (2018). Chemical composition and assessment of larvicidal and repellent capacity of 14 Lamiaceae essential oils against Aedes albopictus. Parasitol. Res. 117, 1953-1964. doi: 10.1007/s00436-018$5892-9$

Gonzalez-Coloma, A., Diaz, C. E., Julio, L. F., Burilo, J., and Andres, M. F. (2017). "A case study of MAPs production. uses and commercialization of Artemisia absinthium Var. candial: extract characterization and valorization," in Frontiers in Horticulture Medicinal and Aromatic Plants: The Basics of Industrial Application Vol. 1, ed M. P. Arraiza (Sharjah: Bentham Science), 163-196. doi: 10.2174/97816810855001170 10014

Goode, P., Ellse, L., and Wall, R. (2018). Preventing tick attachment to dogs using essential oils. Ticks Tick-Borne Dis. 9, 921-926. doi: 10.1016/j.ttbdis.2018.03.029

Grandi, G., Chitimia-Dobler, L., Choklikitumnuey, P., Strube, C., Springer, A., et al. (2020). First records of adult Hyalomma marginatum and $\mathrm{H}$. rufipes ticks (Atari: Ixodidae) in Sweden. Ticks Tick-Borne Dis. 11:101403. doi: 10.1016/j.ttbdis.2020.101403

Gregory, P. J., Johnson, S. N., Newton, A. C., and Ingram, J. S. I. (2009). Integrating pests and pathogens into the climate change/food security debate. J. Exp. Bot. 60, 2827-2838. doi: 10.1093/jxb/erp080

Grul'ová, D., Mudrončeková, S., Zheljazkov, V. D.,Šalamon, I., and Rondon, S.I. (2017). Effect of plant essential oils against Rophalosiphum padi on wheat and barley. Nat. Prod. Commun. 12, 1381-1528. doi: 10.1177/1934578X1701200933

György, Z., Incze, N., and Pluhár, Z. (2020). Differentiating Thymus vulgaris chemotypes with ISSR molecular markers. Biochem. Syst. Ecol. 92:104118. doi: $10.1016 /$ j.bse.2020.104118

Hansford, K. M., Carter, D., Gillingham, E. L., Hernandez-Triana, L. M., Chamberlain, J., Cull, B., et al. (2019). Hyalomma rufipes on an untraveled horse: is this the first evidence of Hyalomma nymphs successfully moulting in the United Kingdom? Ticks Tick Borne Dis. 10, 704-708. doi: 10.1016/j.ttbdis.2019.03.003

Ikbal, C., and Pavela, R. (2019). Essential oils as active ingredients of botanical insecticides against aphids. J. Pest Sci. 92, 971-986. doi: 10.1007/s10340-019-01089-6

Irfan, A., Isa, T., and Oender, C. (2009). Toxicity of essential oil vapours obtained from several plants species against the granary weevil, Sitophilus granarius (L.). Fresen. Environ. Bull. 18, 1717-1722.

Isman, M. B. (2020a). Botanical insecticides in the twenty-first century-fulfilling their promise? Annu. Rev. Entomol. 65, 233-249. doi: 10.1146/annurev-ento-011019-025010

Isman, M. B. (2020b). Commercial development of plant essential oils and their constituents as active ingredients in bioinsecticides. Phytochem. Rev. 19, 235-241. doi: 10.1007/s11101-019-09653-9

Kasrati, A., Alaoui Jamali, C., Bekkouche, K., Spooner-Hart, R., Leach, D., and Abbad, A. (2015). Chemical characterization and insecticidal properties of essential oils from different wild populations of Mentha suaveolens subsp. timija (Briq.) harley from Morocco. Chem. Biodivers. 12, 823-831. doi: $10.1002 / \mathrm{cbdv} .201400236$

Kedia, A., Bhanu, P., and Mishra, P. K. (2014). Antifungal, antiaflatoxigenic, and insecticidal efficacy of spearmint (Mentha spicata L.) essential oil. Int. Biodeterior. Biodegr. 89, 29-36. doi: 10.1016/j.ibiod.2013.10.027

Kimbaris, A. C., González-Coloma, A., Andrés, M. F., Vidali, V. P., Polissiou, M. G., and Santana-Méridas, O. (2017). Biocidal compounds from Mentha sp. essential oils and their structure-activity relationships. Chem. Biodivers. 14:e1600270. doi: 10.1002/cbdv.201600270

Kumar, V., and Tyagi, D. (2013). Chemical composition and biological activities of essential oils of genus Tanacetum-a review. J. Pharmacogn. Phytochem. $2,159-163$

Laghzaoui, E., Abderrafea, E., Ayoub, K., Abdelaziz, A., and El Hassan, E. M. (2019). Toxicity of essential oils obtained from Juniperus thurifera var. africana and Mentha suaveolens subsp. timija chemotypes against preadult stages of Hyalomma aegyptium tick (Acari: Ixodidae). Nat. Prod. Res. doi: $10.1080 / 14786419.2019 .1677658$ 
Li, X., He, T., Wang, X., Shen, M., Yan, X., Fan, S., et al. (2019). Traditional uses, chemical constituents and biological activities of plants from the genus Thymus. Chem. Biodivers. 16:e1900254. doi: 10.1002/cbdv.201900254

Lotfi, D., and Karima, K. (2020). Identification and incidence of hard tick species during summer season 2019 in Jijel Province (northeastern Algeria). J. Parasit. Dis. 45, 211-217. doi: 10.1007/s12639-020-01296-4

Lukas, B., Schmiderer, C., and Novak, J. (2015). Essential oil diversity of European Origanum vulgare L. (Lamiaceae). Phytochem. 119, 32-40. doi: 10.1016/j.phytochem.2015.09.008

Maron, J. L., Agrawal, A. A., and Schemske, D. W. (2019). Plant-herbivore coevolution and plant speciation. Ecology 100:e02704. doi: 10.1002/ecy.2704

Martinez-Velazquez, M., Rosario-Cruz, R., Castillo-Herrera, G., Flores-Fernandez, J. M., Alvarez, A. H., and Lugo-Cervantes, E. (2011). Acaricidal effect of essential oils from Lippia graveolens (Lamiales: Verbenaceae), Rosmarinus officinalis (Lamiales: Lamiaceae), and Allium sativum (Liliales: Liliaceae) against Rhipicephalus (Boophilus) microplus (Acari: Ixodidae). J. Med. Entomol. 48, 822-827. doi: 10.1603/ME10140

Mkolo, M. N., and Magano, S. R. (2007). Repellent effects of the essential oil of Lavendula angustifolia against adults of Hyalomma marginatum rufipes. J. S. Afr. Vet. Assoc. 78, 149-152. doi: 10.4102/jsava.v78i3.307

Mohamed, A. E-H. H., El-Sayed, M. A., Hegazy, M. E., Helaly, S., Esmail, A. M., and Mohamed, N. S. (2010). Chemical constituents and biological activities of Artemisia herba-alba. Rec. Nat. Prod. 4, 1-25.

Morales Valverde, R. (1997). Synopsis of the genus Thymus L. in the mediterranean area. Lagascalia 19, 249-262.

Navarro-Rocha, J., Andrés, M. F., Díaz, C. E., Burillo, J., and GonzálezColoma, A. (2020). Composition and biocidal properties of essential oil from pre-domesticated Spanish Satureja Montana, Ind. Crop. Prod. 145:111958. doi: 10.1016/j.indcrop.2019.111958

Navarro-Rocha, J., Barrero, A. F., Burillo, J., Olmeda, A. S., and GonzálezColoma, A. (2018). Valorization of essential oils from two populations (wild and commercial) of Geranium macrorrhizum L. Ind. Crops Prod. 116, 41-45. doi: 10.1016/j.indcrop.2018.02.046

Ntalli, N., Koliopoulos, G., Giatropoulos, A., and Menkissoglu-Spiroudi, U. (2019). Plant secondary metabolites against arthropods of medical importance. Phytochem. Rev. 18, 1255-1275. doi: 10.1007/s11101-019-09647-7

Nubia, S. V., Oliveira, F., Romero, C., Matos, C., and Helena, C. (2016). Fumigation toxicity of essential oils against Rhyzopertha dominica (F.) in stored maize grain. Revista Caatinga 29, 435-440. doi: 10.1590/1983-21252016v29n220rc

OEPP/EPPO (2015). Diagnostic protocol for Spodoptera littoralis, Spodoptera litura, Spodoptera frugiperda, Spodoptera eridania. Bulletin OEPP/EPPO 45, 410-444. doi: 10.1111/epp.12258

Oerke, E. C. (2006). Crop losses to pests. J. Agric. Sci. 144, 31-43. doi: $10.1017 /$ S0021859605005708

Ortiz de Elguea-Culebras, G., Sánchez-Vioque, R., Berruga, M. I., HerraizPeñalver, D., González-Coloma, A., Andrés, M. F., et al. (2018). Biocidal Potential and Chemical Composition of Industrial Essential Oils from Hyssopus officinalis, Lavandula $\times$ intermedia var. Super, and Santolina chamaecyparissus. Chem. Biodivers. 15:e1700313. doi: 10.1002/cbdv.201700313

Oumzil, H., Ghoulami, S., Rhajaoui, M., Ilidrissi, A., Fkih-Tetouani, S., Faid, M., et al. (2002). Antibacterial and antifungal activity of essential oils of Mentha suaveolens. Phytother. Res. 16, 727-731. doi: 10.1002/ptr.1045

Park, C. G., Jang, M. K., Yoon, A., and Kim, J. (2016). Insecticidal and acetylcholinesterase inhibitory activities of Lamiaceae plant essential oils and their major components against Drosophila suzukii (Diptera: Drosophilidae). Ind. Crops Prod. 89, 507-513. doi: 10.1016/j.indcrop.2016.06.008

Pavela, R. (2005). Insecticidal activity of some essential oils against larvae of Spodoptera littoralis. Fitoterapia 76, 691-696. doi: 10.1016/j.fitote.2005.06.001

Pavela, R. (2011). Antifeedant and larvicidal effects of some phenolic components of essential oils lasp lines of introduction against Spodoptera littoralis (Boisd.). J. Essent. Oil-Bear. Plants 14, 266-273. doi: 10.1080/0972060X.2011.10643932

Pavela, R. (2012). Sublethal effects of some essential oils on the cotton leafworm Spodoptera littoralis (Boisduval). J. Essent. Oil-Bear. Plants 15, 144-156. doi: 10.1080/0972060X.2012.10644030

Pavela, R. (2014). Acute, synergistic and antagonistic effects of some aromatic compounds on the Spodoptera littoralis Boisd. (Lep., Noctuidae) larvae. Ind. Crops Prod. 60, 247-258. doi: 10.1016/j.indcrop.2014.06.030
Pavela, R. (2016). History, presence and perspective of using plant extracts as commercial botanical insecticides and farm products for protection against insects - a review. Plant Protect. Sci. 52, 229-241. doi: 10.17221/31/2016-PPS

Pavela, R., and Benelli, G. (2016). Essential oils as ecofriendly biopesticides? Challenges and constraints. Trends Plant Sci. 21, 1000-1007. doi: 10.1016/j.tplants.2016.10.005

Pavela, R., Kaffková, K., and Kumšta, M. (2014). Chemical composition and larvicidal activity of essential oils from different Mentha L. and Pulegium species against Culex quinquefasciatus Say (Diptera: Culicidae). Plant Prot. Sci. 50, 36-41. doi: 10.17221/48/2013-PPS

Pérez-Sánchez, R., Ubera, J. L., Lafont, F., and Gálvez, C. (2008). Composition and variability of the essential oil in Thymus zygis from Southern Spain. J. Essent. Oil Res. 20, 192-200. doi: 10.1080/10412905.2008.9699989

Petrakis, E. A., Kimbaris, A. C., Perdikis, D. C., Lykouressis, D. P., Tarantilis, P. A., and Polissiou, M. G. (2014). Responses of Myzus persicae (Sulzer) to three Lamiaceae essential oils obtained by microwaveassisted and conventional hydrodistillation. Ind. Crops Prod. 62, 272-279. doi: 10.1016/j.indcrop.2014.08.041

Picard, I., Hollingsworth, R. G., Salmieri, S., and Lacroix, M. (2012). Repellency of essential oils to Frankliniella occidentalis (Thysanoptera: Thripidae) as affected by type of oil and polymer release. J. Econ. Entomol. 105, 1238-1247. doi: 10.1603/EC11292

Pirali-Kheirabadi, K., and Teixeira da Silva, J. A. (2010). Lavandula angustifolia essential oil as a novel and promising natural candidate for tick (Rhipicephalus (Boophilus) annulatus) control. Exp. Parasitol. 126, 184-186. doi: 10.1016/j.exppara.2010.04.012

Püntener, W. (1981). Manual for Field Trials in Plant Protection. 2nd Edn. Agricultural Division. Basle, Switzerland: Ciba-Geigy.

Regnault-Roger, C., Vincent, C., and Arnason, J. T. (2012). Essential oils in insect control: low-risk products in a high-stakes world. Annu. Rev. Entomol. 57, 405-425. doi: 10.1146/annurev-ento-120710-100554

Rueden, C. T., Schindelin, J., Hiner, M. C., DeZonia, B. E., Walter, A. E., Arena, E. T., et al. (2017). ImageJ2: ImageJ for the next generation of scientific image data. BMC Bioinformatics 18:529. doi: 10.1186/s12859-017-1934-Z

Ruiz-Vásquez, L., Olmeda, A. S., Zúñiga, G., Villarroel, L., Echeverri, L., González-Coloma, A., et al. (2017). Insect antifeedant and ixodicidal compounds from Senecio adenotrichius. Chem. Biodivers. 14:e1600155. doi: 10.1002/cbdv.201600155

Salido, S., Valenzuela, L. R., Altarejos, J., Nogueras, M., Sánchez, A., and Cano, E. (2004). Composition and infraspecific variability of Artemisia herba-alba from southern Spain. Biochem. Syst. Ecol. 32, 265-277. doi: 10.1016/j.bse.2003.09.002

Salman, M., Abbas, R. Z., Israr, M., Abbas, A., Mehmood, K., Khan, M. K., et al. (2020). Repellent and acaricidal activity of essential oils and their components against Rhipicephalus ticks in cattle. Vet. Parasitol. 283:109178. doi: 10.1016/j.vetpar.2020.109178

Sangha, J. S., Astatkie, T., and Cutler, G. C. (2017). Ovicidal, larvicidal, and behavioural effects of some plant essential oils on diamondback moth (Lepidoptera: Plutellidae). Can. Entomolo. 149, 639-648. doi: $10.4039 /$ tce. 2017.13

Santana, O., Andrés, M. F., Sanz, J., Errahmani, N., Abdeslam, L., and GonzálezColoma, A. (2014). Valorization of essential oils from Moroccan aromatic plants. Nat. Prod. Commun. 9, 1109-1114. doi: 10.1177/1934578X1400900812

Saroukolai, A. T., Nouri-Ganbalani, G., and Rafiee-Dastjerdi, H. (2014). Antifeedant activity and toxicity of some plant essential oils to colorado potato beetle, Leptinotarsa decemlineata Say (Coleoptera: Chrysomelidae). Plant Prot. Sci. 50, 207-216. doi: 10.17221/9/2014-PPS

Silva, F. V. M., Martins, A., Salta, J., Neng, N. R., Nogueira, J. M. F., Mira, D., et al. (2009). Phytochemical profile and anticholinesterase and antimicrobial activities of supercritical versus conventional extracts of Satureja montana. J. Agric. Food Chem. 57, 11557-11563. doi: 10.1021/jf901786p

Singh, P., and Pandey, A. K. (2018). Prospective of essential oils of the genus Mentha as biopesticides: a review. Front. Plant Sci. 9:1295. doi: 10.3389/fpls.2018.01295

Soutar, O., Cohen, F., and Wall, R. (2019). Essential oils as tick repellents on clothing. Exp. Appl. Acarol. 79, 209-219. doi: 10.1007/s10493-019-00422-z

Štefanidesová, K., Škultéty, L., Sparagano, O.A.E., and Špitalská, E. (2017). The repellent efficacy of eleven essential oils against adult Dermacentor 
reticulatus ticks. Ticks Tick Borne Dis. 8, 780-786. doi: 10.1016/j.ttbdis.2017. 06.003

Tabari, M. A., Youssefi, M. R., Maggi, F., and Benelli, G. (2017). Toxic and repellent activity of selected monoterpenoids (thymol, carvacrol and linalool) against the castor bean tick, Ixodes ricinus (Acari: Ixodidae). Vet. Parasitol. 245, 86-91. doi: 10.1016/j.vetpar.2017.08.012

Tepe, B., and Cilkiz, M. (2016). A pharmacological and phytochemical overview on Satureja. Pharm. Biol. 54, 375-412. doi: 10.3109/13880209.2015.1043560

Usanmaz-Bozhuyuk, A., and Kordali, S. (2018). Investigation of the toxicity of essential oils obtained from six Satureja species on Colorado Potato Beetle, Leptinotarsa decemlineata (Say, 1824), (Coleoptera: Chrysomelidae). Fresen. Environ. Bull. 27, 4389-4401. doi: 10.30616/ajb.623827

van Emden, H. F., and Harrington, R. (2017). Aphids as Crop Pests. Oxfordshire: CABI, 717. doi: 10.1079/9781780647098.0000

Velasco, A., and Perez-Alonso, M. J. (1983). Estudio químico del aceite esencial de diversas "Saturejae" Ibericas. An. Jard. Bot. Madr. 40, 107-118.
Zekri, N., Sabri, H., Khannouchi, S., El Belghiti, M. A., and Zair, T. (2013). Phytochemical study and fumigant toxicity of Mentha suaveolens Ehrh essential oil from Morocco against adults of S. oryzae (L.). Aust. J. Basic Appl. Sci. 7, 599-606.

Conflict of Interest: The authors declare that the research was conducted in the absence of any commercial or financial relationships that could be construed as a potential conflict of interest.

Copyright (c) 2021 Valcárcel, Olmeda, González, Andrés, Navarro-Rocha and González-Coloma. This is an open-access article distributed under the terms of the Creative Commons Attribution License (CC BY). The use, distribution or reproduction in other forums is permitted, provided the original author(s) and the copyright owner(s) are credited and that the original publication in this journal is cited, in accordance with accepted academic practice. No use, distribution or reproduction is permitted which does not comply with these terms. 